\title{
Resolving spatial heterogeneity of noble gas isotopes in chondrites using laser ablation
}

\author{
DAVID J BYRNE ${ }^{1}$, MICHAEL W BROADLEY ${ }^{1}$, \\ MATTHIEU ALMAYRAC ${ }^{2}$ AND BERNARD MARTY ${ }^{3}$ \\ ${ }^{1}$ Centre de Recherches Pétrographiques et Géochimiques \\ ${ }^{2}$ CRPG Université de Lorraine \\ ${ }^{3}$ Université de Lorraine \\ Presenting Author: david.byrne@univ-lorraine.fr
}

The noble gas isotopic composition of meteorites provides information on the distribution of volatiles in the early solar system, and their delivery during planetary formation [1]. The distribution of cosmogenic noble gas isotopes can give key insights into the irradiation history of the meteorite parent body, as well as any pre-irradiation recorded in the accretionary phases [2].

However, due to the low abundance of the noble gases, relatively large sample sizes are required for analysis. Conventional analysis techniques such as step-heating obscure the heterogeneity between the plethora of different phases within meteorites, especially chondrites. We present a new extraction system developed at CRPG Nancy, utilising laser ablation combined with traditional noble gas mass spectrometry. This allows in-situ targeting of chondrite thick sections, permitting easy resolution of isotopic signatures in chondrules, rims, CAI's and matrix. Ablation pit volumes are calculated using a laser interferometer for determination of noble gas abundances.

We present preliminary data measured in the Paris $\mathrm{CM}$ chondrite, Acfer $082 \mathrm{CV}$ chondrite, and Erg Chech 002 andesitic achondrite [3]. In each sample several different phases were selected for analysis, and isotopes of $\mathrm{He}, \mathrm{Ne}$ and $\mathrm{Ar}$ were measured. Isotopic signatures are dominated by cosmogenic isotopes within chondrules, while matrix analyses record signatures closer to "planetary" isotopic endmembers. Significant differences up to a factor of 5 in cosmogenic exposure levels between chondrules and matrix may be due to pre-irradiation of chondrules before accretion into the chondrite parent body, but could also be due to the small grain sizes in the matrix precluding effective retention of noble gases. Chondrule rims in Acfer 082 show anomalously low ${ }^{21} \mathrm{Ne} /{ }^{22} \mathrm{Ne}$ ratios, which is likely due to increased concentrations of presolar material in the chondrule rim. Signatures of ${ }^{40} \mathrm{Ar} /{ }^{36} \mathrm{Ar}$ and neon isotopes in Erg Chech 002 show variations in the isotopic composition of the olivine and pyroxene xenocrysts, and that of the fine-grained groundmass.

We would like to thank Jean-Alix Barrat and Addi Bischoff for the provision of the samples used in this study.

[1] Ott, 2014, Geochemistry 74:4

[2] Riebe et al., 2017, GCA 213

[3] Barratt et al., 2021, PNAS (in press) 